\title{
Population-Based Age Group Specific Annual Incidence Rates of Symptomatic Age-Related Macular Degeneration
}

\author{
Jukka M. Saari* \\ Department of Ophthalmology, Helsinki University Eye and Ear Hospital, Helsinki, and Department of Ophthalmology, \\ Central Hospital of Central Finland, Jyväskylä, Finland
}

\begin{abstract}
Purpose. To study the population-based annual incidence rates of exudative, dry and all cases of symptomatic age-related macular degeneration (AMD) in different age and sex groups.

Methods. This is a one year, prospective, population-based study on all consecutive new patients with AMD in the hospital district of Central Finland. The diagnosis was confirmed in all patients with slit lamp biomicroscopy, optical coherence tomography (OCT) using a Spectralis HRA + OCT device, and the Heidelberg Eye Explorer 1.6.2.0 program. Fluorescein angiograms were taken when needed.

Results. The population-based annual incidence rates of all cases of symptomatic AMD increased from $0.03 \%$ (95\% CI, $0.01-0.05 \%$ ) in the age group $50-59$ years to $0.82 \%(95 \% \mathrm{CI}, 0.55-1.09 \%)$ in the age group $85-89$ years and were $0.2 \%$ (95\% CI, 0.17-0.24\%) in exudative, $0.11 \%$ (95\% CI, 0.09-0.14\%) in dry, and $0.32 \%$ (95\% CI, $0.28-0.36 \%)$ in all cases of AMD in the age group 60 years and older. During the next 20 years in Central Finland the population-based annual incidence rates can be estimated to increase to $0.27 \%$ (95\% CI, $0.24-0.30 \%)$ in exudative, to $0.13 \%(95 \% \mathrm{CI}, 0.11-0.15 \%)$ in dry, and to $0.41 \%(95 \% \mathrm{CI}, 0.37-0.45 \%)$ in all cases of AMD in the age group 60 years and older. The populationbased annual incidence of AMD did not show statistically significant differences between males and females $(p>0.1)$.

Conclusion: The population-based age-group specific annual incidence rates of symptomatic AMD of this study may help to plan health care provision for patients of AMD.
\end{abstract}

Keywords: Age-related macular degeneration, incidence, macula, optical coherence tomography, retina.

\section{INTRODUCTION}

Age-related macular degeneration (AMD) is the leading cause of visual impairment and blindness in older people in industrial countries $[1,2]$. While treatment for dry AMD remains to be established, treatment for exudative AMD using anti-vascular endothelial growth factors (anti-VEGF) is being increasingly used to limit progression of the condition [3, 4]. In order to plan adequate healthcare provision, estimates of incidence of especially exudative AMD are required $[4,5]$. Large population-based cohort studies by using grading of fundus photographs showed that the incidence of AMD increased with age [6-9]. Some recent studies used literature search to obtain prevalence estimates of AMD [10-12], and the prevalence estimates were used to estimate annual incidence of AMD [11]. There is only one earlier study showing an average number of exudative AMD in a defined population [4].

Posterior segment optical coherence tomography (OCT) is a non-invasive imaging modality that can quantitatively and qualitatively detect and record retinal pathological signs not seen with other imaging modalities [13-15]. It is suggested for patients with decreased visual acuity,

*Address correspondence to this author at the Runeberginkatu 35.A.4, 00100 Helsinki, Finland; Tel: +358 405077479; Fax: +358 9471 73163;

E-mail: jmsaari@gmail.com metamorphopsia or a central scotoma, and is considered mandatory in the diagnostics of AMD [16]. In Finland, the centralised eye service in every central hospital district with their contained population, and the rule that the patients have to go for treatment in their local central hospital, provide an opportunity to gauge the incidence and service load of different eye diseases. This study describes the populationbased annual incidence rates of exudative, dry and all cases of symptomatic AMD in different age and sex groups confirmed by OCT examination in Central Finland.

\section{MATERIALS AND METHODOLOGY}

The study design was approved by the Ethics Committee of the Central Hospital of Central Finland. The study was conducted in accordance with the ethical standards laid down in the 1964 Declaration of Helsinki, as renewed in 2008.

This is a prospective study of all consecutive new patients examined during a one year period between December $18^{\text {th }} 2008$ and December $17^{\text {th }} 2009$ due to symptomatic AMD in the Central Hospital of Central Finland. The Central Hospital of Central Finland was the only clinic offering OCT examination and treatment for exudative and other advanced AMD in 2009 in the area. The private clinics in this area referred the AMD patients for OCT examination and treatment to the Central Hospital in 2009. The coverage of specialists for the population (one ophthalmologist for every about 8000 persons) is good in the 
hospital district of Central Finland. It is reasonable to assume that the majority of the people with symptoms from AMD in the hospital district of Central Finland were able to contact a doctor and were referred to the Central Hospital of Central Finland for diagnosis and treatment.

The diagnosis of AMD was confirmed with stereoscopic slit-lamp biomicroscopy using a contact lens, OCT, and if needed, by fluorescein angiography. Cases were classified as exudative and dry AMD by using the criteria defined by the International Age-related Maculopathy Epidemiological Study Group [17]. Patients with early age-related maculopathy [17] and all non-AMD maculopathies were excluded from the material. Similarly all patients with AMD diagnosed and treated before the study period were excluded from the material. Only patients with residence in the hospital district of Central Finland were included in this study.

The Central Hospital of Central Finland had the only OCT device in the hospital district, and all patients with symptomatic AMD in the area of Central Finland were referred to the Central Hospital. The hospital record analysis and the database of the OCT device provided a complete collection of all new AMD cases in the hospital district area. OCT and fluorescein angiographic images were taken with Spectralis HRA + OCT device (Heidelberg engineering GmbH, Tiergartenstrasse 15, D-69121 Heidelberg, Germany). The data was analysed using the Heidelberg Eye Explorer 1.6.2.0 program.

The hospital district of Central Finland comprises 23 municipalities in Central Finland. The combined population of the hospital district was 272784 people as of December 2009 [18]. The age and sex distribution of the population in the hospital district during the year 2009 (Table 1) shows that the population aged 50 years or older comprised 107812 people or $40 \%$ of the whole population including $14 \%$ in the age group 50-59 years, $8 \%$ in the age group $60-64$ years, $5 \%$ in the age group 65-69 years, and $13 \%$ in the age group 70 years or older.

Data was collected and analysed with Excel 2007 (Microsoft Corporation, One Microsoft Way, Redmond, WA 98052-6399). The population based annual incidence rates were calculated by using corrected population statistics of the hospital district of the Central Hospital of Central Finland for the year 2009 (Table 1). The 95\% confidence intervals $(95 \% \mathrm{CI})$ for the population-based annual incidence rates of AMD were calculated using normal approximation of Poisson distribution. The differences in population-based age group specific annual incidence rates of AMD between genders were separately tested by Pearson's chi-square test.

The Population Registration Centre of the Central Statistical Office of Finland published official statistics of
Finland with population projection for years $2009-2060$ including the population statistics for different age groups of the area of Central Finland [19]. By using the population projection statistics of different age groups in Central Finland in 2020 and 2030 [19] and the population-based age group specific annual incidence rates of exudative, dry and of all symptomatic AMD observed in the present study (Tables 2-4) I estimated the comparative annual incidence rates of AMD in Central Finland in 2020 and 2030.

\section{RESULTS}

In total, 231 patients with symptomatic AMD, 91 males $(39 \%)$ and 140 females $(61 \%)$ with a mean age of $76.8 \pm 9.1$ years (median 80 , range $50-95$ ); in males $76.0 \pm 9.4$ years, in females $78.6 \pm 7.8$ years were enrolled in the study. The population-based annual incidence of all symptomatic AMD patients was $0.03 \%(95 \% \mathrm{CI}, 0.01-0.05 \%)$ in the age group $50-59$ years, it rose with the age to $0.82 \%(95 \% \mathrm{CI}, 0.55$ $1.09 \%$ ) in the age group $85-89$ years, and thence declined to $0.63 \%(95 \% \mathrm{CI}, 0.24-1.02 \%)$ in the age group 90 years and older (Table 2). It was $0.21 \%$ (95\% CI, $0.18-0.24 \%$ ) in all AMD patients 50 years or older and $0.32 \%$ (95\% CI, 0.28 $0.36 \%$ ) in all AMD patients 60 years or older.

In the total material there were 146 patients, 57 males and 89 females with exudative AMD (63.2\% of all cases). The population-based annual incidence of exudative AMD was $0.02 \%(95 \% \mathrm{CI}, 0-0.03 \%)$ in the age group $50-59$ years and increased with the age to $0.65 \%$ (95\% CI, 0.47-0.83\%) in the age group $80-84$ years, with a decline beyond 85 years of age (Table 3). It was $0.14 \%(95 \% \mathrm{CI}, 0.11-0.16 \%)$ in the age group 50 years or older, and $0.20 \%(95 \% \mathrm{CI}, 0.17$ $0.24 \%$ ) in the age group 60 years and older

In the total material there were 85 patients, 34 males and 51 females with dry AMD (36.9\% of all cases). The annual incidence of dry AMD was $0.02 \%(95 \% \mathrm{CI}, 0-0.03 \%)$ in the age group 50-59 years, and increased with age to $0.36 \%$ (95\% CI, $0.18-0.54 \%)$ in the age group 85-89 years, with a decline beyond 90 years of age (Table 4). It was $0.08 \%(95 \%$ $\mathrm{CI}, 0.06-0.1 \%)$ in the age group 50 years and older, and $0.11 \%(95 \%$ CI $0.09-0.14 \%)$ in the age group 60 years and older.

In the hospital district of Central Finland the ratio of males-females was 1:1.2 in the age group 50 years and older and 1:1.3 in the age group 60 years and older (Table 1). In all patients with symptomatic AMD (Table 2) the ratio of males-females was 1:1.5 in the age group 50 years and older and 1:1.7 in the age group 60 years and older (Table 2). In males both in patients with exudative and dry AMD, and in females with dry AMD, the population-based annual incidence rates increased from the age group 50-59 years to

Table 1. Age and sex distribution of population in hospital district of Central Finland in 2009.

\begin{tabular}{|c|c|c|c|c|c|c|c|c|c|c|}
\hline \multirow{2}{*}{ Gender } & \multicolumn{10}{|c|}{ Age Groups (Years) } \\
\cline { 2 - 20 } & $\mathbf{0 - 4 9}$ & $\mathbf{5 0 - 5 9}$ & $\mathbf{6 0 - 6 4}$ & $\mathbf{6 5 - 6 9}$ & $\mathbf{7 0 - 7 4}$ & $\mathbf{7 5 - 7 9}$ & $\mathbf{8 0 - 8 4}$ & $\mathbf{8 5 - 8 9}$ & $\mathbf{9 0 -}$ & Total \\
\hline \hline Male & 84425 & 19828 & 10135 & 6450 & 5496 & 4096 & 2630 & 1160 & 355 & 134575 \\
\hline Female & 80547 & 19304 & 10187 & 6866 & 6565 & 5624 & 4908 & 2974 & 1234 & 138209 \\
\hline Total & 164972 & 39132 & 20322 & 13316 & 12061 & 9720 & 7538 & 4134 & 1589 & 272784 \\
\hline
\end{tabular}


Table 2. Number of all cases $(\mathrm{N})$ and population-based annual incidence rates $(\%)$ with confidence intervals $(95 \% \mathrm{CI})$ of symptomatic age-related macular degeneration in different age and sex groups.

\begin{tabular}{|c|c|c|c|c|c|c|}
\hline \multirow{2}{*}{ Age Group (Years } & \multicolumn{2}{|c|}{ Males } & \multicolumn{2}{|c|}{ Females } & \multicolumn{2}{|c|}{ Total } \\
\hline & $\mathbf{N}$ & $\%$ & $\mathbf{N}$ & $\%$ & $\mathbf{N}$ & $\%$ \\
\hline $50-59$ & 9 & $0,05(0.02-0.08)$ & 4 & $0.02(0.001-0.04)$ & 13 & $0.03(0.01-0.05)$ \\
\hline $60-64$ & 7 & $0.07(0.02-0.12)$ & 7 & $0.07(0.02-0.12)$ & 14 & $0.07(0.03-0.11)$ \\
\hline $70-74$ & 15 & $0.27(0.13-0.41)$ & 17 & $0.26(0.14-0.38)$ & 32 & $0.27(0.18-0.36)$ \\
\hline $75-79$ & 18 & $0.44(0.24-0.64)$ & 30 & $0.53(0.34-0.72)$ & 48 & $0.49(0.35-0.63)$ \\
\hline $80-84$ & 18 & $0.68(0.37-0.99)$ & 43 & $0.88(0.62-1.14)$ & 61 & $0.81(0.61-1.01)$ \\
\hline $85-89$ & 13 & $1.12(0.51-1.73)$ & 21 & $0.71(0.41-1.01)$ & 34 & $0.82(0.55-1.09)$ \\
\hline
\end{tabular}

Table 3. Number of cases $(\mathrm{N})$ and population-based annual incidence rates (\%) with confidence intervals $(95 \% \mathrm{CI})$ of exudative age-related macular degeneration in different age and sex groups.

\begin{tabular}{|c|c|c|c|c|c|c|}
\hline \multirow[t]{2}{*}{ Age Group (Years) } & \multicolumn{2}{|c|}{ Incidence $(95 \% \mathrm{CI})$} & \multicolumn{2}{|c|}{ Incidence $(95 \% \mathrm{CI})$} & \multicolumn{2}{|c|}{ Incidence $(95 \%$ CI $)$} \\
\hline & $\mathbf{N}$ & $\%$ & $\mathbf{N}$ & $\%$ & $\mathbf{N}$ & $\%$ \\
\hline $50-59$ & 3 & $0,02(0-0.03)$ & 3 & $0.02(0-0.03)$ & 6 & $0.02(0-0.03)$ \\
\hline $60-64$ & 1 & $0.01(0-0.03)$ & 3 & $0.03(0-0.06)$ & 4 & $0.02(0-0.04)$ \\
\hline $70-74$ & 10 & $0.18(0.07-0.29)$ & 11 & $0.17(0.07-0.27)$ & 21 & $0.17(0.10-0.25)$ \\
\hline $75-79$ & 12 & $0.29(0.12-0.46)$ & 19 & $0.34(0.19-0.49)$ & 31 & $0.32(0.21-0.43)$ \\
\hline $80-84$ & 17 & $0.65(0.34-0.96)$ & 32 & $0.65(0.43-0.88)$ & 49 & $0.65(0.47-0.83)$ \\
\hline $85-89$ & 8 & $0.69(0.21-1.17)$ & 11 & $0.37(0.15-0.59)$ & 19 & $0.46(0.25-0.67)$ \\
\hline
\end{tabular}

the age group 85-89 years, with a decline after the age of 90 years (Tables 3 and $\mathbf{4}$ ). In females with exudative AMD the decline was seen beyond the age of 85 years (Table 3 ). In patients with exudative AMD there was rather even amount of males and females in the age groups 50-59 years to 70-74 years but beyond the age 75 years women had a distinct majority (Table 3). In patients with dry AMD males showed a slight majority in the age groups 50-59 to 65-69 years but beyond the age 75 years females showed majority (Table 4). However, the population- based annual incidence rates of symptomatic AMD in different age groups did not show statistically significant differences between males and females $(\mathrm{p}>0.1)$.

The population-based annual incidence rates were estimated to be $0.22 \%$ (95\% CI, $0.19-0.25 \%)$ in exudative, $0.12 \%(95 \% \mathrm{CI}, 0.09-0.14 \%)$ in dry, and $0.35 \%(95 \% \mathrm{CI}$,
$0.31-0.39 \%)$ in all symptomatic cases of AMD in 2020, and $0.27 \%(95 \% \mathrm{CI}, 0.24-0.30 \%)$ in exudative, $0.13 \%(95 \% \mathrm{CI}$, $0.11-0.16 \%)$ in dry, and $0.41 \%$ (95\% CI, $0.37-0.45 \%)$ in all symptomatic cases of AMD in 2030 in Central Finland.

\section{DISCUSSION}

This is the first study to show actual numbers and age group specific annual incidence rates of exudative, dry and of all cases of symptomatic AMD in different age and sex groups in a whole population of a defined area. Good coverage of referring ophthalmologists, easy availability for the treatment, and confirmation of the diagnosis of every patient by using OCT may indicate that the population-based age group specific annual incidence rates of this study are useful for guiding health service provision for those with AMD. The population-based age group specific annual 
Table 4. Number of cases $(\mathrm{N})$ and population-based annual incidence rates (\%) with confidence intervals $(95 \% \mathrm{CI})$ of dry agerelated macular degeneration in different age and sex groups.

\begin{tabular}{|c|c|c|c|c|c|c|}
\hline \multirow{2}{*}{ Age Group (Years) } & \multicolumn{2}{|c|}{ Males } & \multicolumn{2}{|c|}{ Females } & \multicolumn{2}{|c|}{ Total } \\
\hline & $\mathbf{N}$ & $\%$ & $\mathbf{N}$ & $\%$ & $\mathbf{N}$ & $\%$ \\
\hline $50-59$ & 6 & $0,03(0.01-0.05)$ & 1 & $0.005(0-0.02)$ & 7 & $0.02(0-0.03)$ \\
\hline $60-64$ & 6 & $0.06(0.01-0.11)$ & 4 & $0.04(0-0.08)$ & 10 & $0.05(0.02-0.08)$ \\
\hline $70-74$ & 5 & $0.09(0.01-0.17)$ & 6 & $0.09(0.02-0.16)$ & 11 & $0.09(0.04-0.15)$ \\
\hline $75-79$ & 6 & $0.15(0.03-0.26)$ & 11 & $0.20(0.08-0.31)$ & 17 & $0.17(0.09-0.26)$ \\
\hline $80-84$ & 1 & $0.04(0-0.11)$ & 11 & $0.22(0.09-0.35)$ & 12 & $0.16(0.07-0.25)$ \\
\hline $85-89$ & 5 & $0.43(0.05-0.81)$ & 10 & $0.34(0.13-0.56)$ & 15 & $0.36(0.18-0.54)$ \\
\hline
\end{tabular}

incidence rates of this study were slightly higher than those calculated by examining stereoscopic fundus photographs in the 6.5 years follow-up cohort study in Rotterdam (6).

The population of different age groups may be of different size in different geographic areas and during different times. Due to a big baby boom during the years 1945 to 1949 in Finland this big age group comprised in 2009 the age group 60-64 years. Thus in this study in 2009 the population of the age group 60-69 years consisted $49 \%$ of the population of the age group 60 years and older. During coming years the effect of the big age group on the population-based annual incidence of AMD in the age group 60 years and older can be estimated by using the population projection data of the Official Statistics of Finland [19]

and the population-based age group specific annual incidence rates of AMD of the present study (Tables 2-4). The population-based annual incidence of all symptomatic AMD cases was $0.32 \%(95 \%$ CI, $0.28-0.36 \%)$ in the population aged 60 years and older in this study, and it can be estimated to be in Central Finland $0.35 \%(95 \%$ CI, 0.31 $0.39 \%$ ) in 2020 and $0.41 \%$ (95\% CI, $0.37-0.45 \%)$ in 2030 .

Geirsdottir et al. (4) observed in Iceland (320000 inhabitants) on average 147 patients of exudative AMD per year giving an annual incidence of $0.29 \%$ in the population of about 50000 persons aged 60 years and older. In this study in Central Finland (272784 persons) there were 140 new patients of exudative AMD during one year among the 68680 persons aged 60 years and older $(0.2 \%)$. The big age group of 60-64 years with a small age group specific incidence of exudative AMD (Table 3) may explain why the population-based annual incidence of exudative AMD in the age group 60 years and older was somewhat lower in this study than observed in Iceland. The population-based annual incidence of exudative AMD in the age group 60 years and older can be estimated to be $0.22 \%(95 \% \mathrm{CI}, 0.19-0.25 \%)$ in 2020 and $0.27 \%(95 \%$ CI, 0.24-0.30\%) in 2030 in Central Finland. Aging of the population in Finland during the next 10-20 years may produce increasing demands for health care services not only for patients with AMD but also for treatment of cataract and glaucoma.

Klein et al. observed in the Beaver Dam Eye Study, that the fifteen-year cumulative incidence of early age-related maculopathy with the presence of either soft indistinct drusen or the presence of pigmentary abnormalities together with any type of drusen was about five times higher than that of late AMD [9]. Patients with early age-related maculopathy [17] were excluded from the present material. In this study exudative AMD comprised 63\% and dry AMD $37 \%$ of all symptomatic AMD cases. This is in good agreement with the prevalence studies indicating that exudative AMD accounts $50-83 \%$ of late AMD cases depending on population $[11,20-22]$. The population-based annual incidence of late dry AMD in this study was $0.11 \%$ $(95 \% \mathrm{CI}, 0.09-0.14 \%)$ in the age group 60 years and older, and it can be estimated to increase to $0.12 \%(95 \% \mathrm{CI}, 0.09$ $0.14 \%$ ) in 2020 and to $0.13 \%$ (95\% CI, $0.11-0.16 \%$ ) in 2030 in Central Finland.

Women live longer than men and were represented in greater number both in the normal population (Table 1) and in exudative, dry, and in all cases of AMD in the age group 75 years and older (Tables 2-4). However, in this study the population-based annual incidence rates of exudative, dry, and of all cases of symptomatic AMD were slightly but not statistically significantly higher in females than in males $(\mathrm{p}>0.1)$. This is in good agreement with earlier studies [7, 9, 22].

The demands of the diagnostics and treatment of AMD give an increasing service load to the eye department. In Central Finland AMD was the most frequent eye disease examined by OCT comprising $40 \%$ of retinal and $29 \%$ of all cases [15]. In this study every patient with suspected AMD was studied by a new Spectralis HRA + OCT with 30 images averaged. OCT examination was successful and had a great effect on the diagnostic quality confirming, changing or eliminating the suspected clinical diagnosis of AMD in $95 \%$ of cases [15]. Private clinics have recently begun to give 
anti-VEGF treatment based on OCT examinations, and this may make it more difficult in the future to collect actual incidence data for AMD from a complete population of Central Finland.

In the anti-VEGF treatment of exudative AMD frequent intravitreal injections and follow-up visits are needed. An OCT-guided study with initial three monthly injections followed by variable dosing regimen showed that visual acuity and OCT findings improved with a mean of 5.6 \pm 2.3 (range 3-13) injections over a period of 12 months [5]. Accordingly, in this study the 146 new patients with exudative AMD should need a mean 818 (range 438-1898) injections per year. Approximately 1200 injections were given in Iceland for the on average 147 exudative AMD patients per year which suits well with the range of the suggested regimen [4].

AMD is a multifactorial disease. Both genetic susceptibility and behavioral and lifestyle factors modify the risk of developing AMD [23]. As seen also in this study, AMD has a strong association with age [4, 6-12, 21-23]. Other risk factors associated with AMD include cigarette smoking, previous cataract surgery, higher body mass index, history of cardiovascular disease, hypertension, diabetes, and higher plasma fibrinogen [21-23]. These common risk factors may occur in different populations. In this study all patients with AMD were Caucasians similarly as most people in Central Finland. No significant differences in late AMD prevalence were seen between whites, Asians, Blacks or Hispanics [22, 24, 25]. Thus the population- based agegroup specific annual incidence rates of symptomatic AMD of this study might be used not only for Caucasian but also for other ethnic populations.

\section{CONCLUSION}

This is the first study to determine the population-based age-group specific annual incidence rates of exudative, dry and of all cases of symptomatic AMD in a whole population of a defined area. The population-based annual incidence rates of all cases of late AMD increased with age from $0.03 \%$ (95\% CI, $0.01-0.05 \%$ ) in the age group $50-59$ years to $0.82 \%(95 \% \mathrm{CI}, 0.55-1.09 \%)$ in the age group $85-89$ years, and declined beyond the age of 90 years. In Central Finland in 2009, the population-based annual incidence rates of late AMD in the age group 60 years and older were $0.20 \%(95 \%$ CI, $0.17-0.24 \%)$ for exudative AMD, $0.11 \%(95 \%$ CI, $0.09-$ $0.14 \%$ ) for dry AMD, and $0.32 \%$ (95\% CI, $0.28-0.36 \%)$ for all cases of symptomatic AMD. The population-based agegroup specific annual incidence rates of symptomatic AMD of this study may help to plan health care provision for patients of AMD.

\section{CONFLICT OF INTEREST}

The author does not have conflict of interest with the submission.

\section{ACKNOWLEDGEMENTS}

I would like to thank Professor K. Matti Saari, MD, PhD, FEBO for reviewing the manuscript. This study was supported in part by a grant from Evald and Hilda Nissi Foundation, Espoo, Finland.
Supported in part by a grant from Evald and Hilda Nissi Foundation, Espoo, Finland.

\section{REFERENCES}

[1] Congdon N, O'Colmain B, Klaver CC, et al. Causes and prevalence of visual impairment among adults in United States. Eye Diseases Prevalence Research Group. Arch Ophthalmol 2004; 122: 477- 85.

[2] Bunce C, Xing W, Wormald R. Causes of blind and partial sight certifications in England and Wales: April 2007- March 2008. Eye 2010; 24: 1692-9.

[3] Wong TY, Liew G, Mitchell P. Clinical update: new treatments for age-related macular degeneration. Lancet 2007; 370: 204-6.

[4] Geirsdottir A, Jonsson O, Thorisdottir S, et al. Population-based incidence of exudative age-related macular degeneration and ranibizumab treatment load. Br J Ophthalmol 2012; 96: 444-7.

[5] Fung AE, Lalwani GA, Rosenfeld PJ, et al. An optical coherence tomography-guided variable dosing regimen with intravitreal ranibizumab (Lucentis) for neovascular age-related macular degeneration. Am J Ophthalmol 2007; 143: 566-83.

[6] van Leeuwen R, Klaver CCW, Vingerling JR, Hofman A, de Jong PTVM. The risk and natural course of age-related maculopathy. Follow-up at 6,5 years in the Rotterdam study. Arch Ophthalmol 2003; 121: 519-26.

[7] Buch H, Nielsen NV, Vinding T, Jensen GB, Prause JU, la Cour M. 14-year incidence, progression, and visual morbidity of age-related maculopathy. The Copenhagen City Eye Study. Ophthalmology 2005; 112: 787-98.

[8] Wang JJ, Rochtchina E, Lee AJ, et al. Ten- year incidence and progression of age-related maculopathy. The Blue Mountains eye study. Ophthalmology 2007; 114: 92-8.

[9] Klein R, Klein BEK, Knudtson MD, Meuer SM, Swift M, Gangnon RE. Fifteen-year cumulative incidence of age-related macular degeneration. The Beaver Dam Eye Study. Ophthalmology 2007; 114: 253-62.

[10] Kawasaki R, Yasuda M, Song SJ, et al. The prevalence of agerelated macular degeneration in Asians. A systemic review and meta-analysis. Ophthalmology 2010; 117: 921-7.

[11] Owen CG, Jarrar Z, Wormald R, Cook DG, Fletcher AE, Rudnicka AR. The estimated prevalence and incidence of late stage agerelated macular degeneration in the UK. Br J Ophthalmol 2012; 96 : 752- 6.

[12] Rudnicka AR, Jarrar Z, Wormald R, Cook DG, Fletcher A, Owen CG. Age and gender variations in age-related macular degeneration prevalence in populations of European ancestry: a meta-analysis. Ophthalmology 2012; 119: 571-80.

[13] Yang Q, Reisman CA, Wang Z, et al. Automated layer segmentation of macular OCT images using dual-scale gradient information. Opt Express 2010; 18: 21293-307.

[14] Geitzenauer W, Hitzenberger CK, Schmidt-Erfurth N. Retinal optical coherence tomography: past, present and future perspectives. Br J Ophthalmol 2011; 95: 171-7.

[15] Saari JM. Diagnostic value of spectral domain optical coherence tomography. J Ophthalmic Photogr 2013; 17-23.

[16] Wakabayashi Y, Nishimura A, Higashide T, Ijiri S, Sugiyama K Unilateral choroidal excavation in the macula detected by spectraldomain optical coherence tomography. Acta Ophthalmol 2010;88: 87-91.

[17]. Bird AC, Bressler NM, Bressler SB, et al. An international classification and grading system for age-related maculopathy and age-related macular degeneration. The International ARM Epidemiological Study Group. Surv Ophthalmol 1995; 39: 367-74.

[18] Finland's Population Register Centre, Central Statistical Office of Finland. Structure of population by municipality 31.12.2009. Helsinki 2010.

[19] Official Statistics of Finland. Population projection 2009-2060. Helsinki 2009.

[20] Krishnan T, Ravindran RD, Murthy GV, et al. Prevalence of early and late age- related macular degeneration in India: the INDEYE study. Invest Ophthalmol Vis Sci 2010; 51: 701-7.

[21] Jonasson F, Arnarsson A, Eiríksdottir G. Prevalence of age-related macular degeneration in old persons: Age, Gene/environment Susceptibility Reykjavik Study. Ophthalmology 2011; 118: 825-30.

[22] Chakravarthy U, Wong TY, Fletcher A, et al. Clinical risk factors for age-related macular degeneration: a systematic review and 
meta-analysis. BMC Ophthalmology 2010; 10: 31: Available from http://www.biomedcentral.com/1471-2415/10/31.

[23] Seddon JM, Reynolds R, Rosner B. Associations of smoking, body mass index, dietary lutein, and genetic variants 10468017 with advanced age-related macular degeneration. Mol Vis 2010; 16: 2412-24.
[24] Klein R, Klein BE, Jensen SC, Maree-Perlman JA, Cruickshanks KJ, Palta M. Age-related maculopathy in a multiracial United States population: The National Health and Nutrition Examination Survey III. Ophthalmology 1999; 106: 1056-65.

[25] Klein R, Klein BE, Knudtson MD, et al. Prevalence of age-related macular degeneration in $4 \mathrm{racial} / \mathrm{ethnic}$ groups in the multi-ethnic study of atherosclerosis. Ophthalmology 2006; 113: 373-80.

(C) Jukka M. Saari; Licensee Bentham Open.

This is an open access article licensed under the terms of the Creative Commons Attribution Non-Commercial License (http://creativecommons.org/licenses/by-nc/3.0/) which permits unrestricted, non-commercial use, distribution and reproduction in any medium, provided the work is properly cited. 